\title{
CONJUGACY CLASSES OF $\Gamma(2)$ AND SPECTRAL RIGIDITY
}

\author{
RALPH PHILLIPS
}

ABstract. The free group $\Gamma(2)$ is generated by $A=(12,01)$ and $B=$ $(10,-21)$, and setting $\chi_{(\xi, \eta)}(A)=\exp (2 \pi i \xi), \chi_{(\xi, \eta)}(B)=\exp (2 \pi i \eta)$ defines a unitary character on $\Gamma(2)$ for $0 \leq \xi, \eta<1$. A program is devised to compute

$$
\mu(\operatorname{tr})=\sum \chi_{(\xi, \eta)}(\text { conj. class) },
$$

summed over all primitive conjugacy classes of $\Gamma(2)$ of trace tr. Combined with a Luo-Sarnak theorem, this yields lower bounds for the spectral variance for a large sampling of characters in the range $0<\xi, \eta<1$. The results indicate that the Berry conjecture for spectral rigidity does not hold for this set of classically chaotic systems. The program is also used to compute

$$
\theta(x)=\sum \ln (N(\{\gamma\})),
$$

summed over all primitive conjugacy classes of $\Gamma(2)$ of norm $N(\{\gamma\}) \leq x$. The function $\theta(x)$ is asymptotic to $x$, and the remainder can be written as $|\theta(x)-x|=x^{\beta}$. The values of $\beta(x)$ are computed for all traces between 3202 and 4802 (here $x=\operatorname{tr}^{2}-2$ ). The $\beta$ 's cluster around 0.6 , attaining a maximum of $2 / 3$. Finally, it is proved that the remainder $\theta(x)-x$ has a negative bias by showing that the mean normalized remainder converges to a negative limit.

\section{INTRODUCTION}

The purpose of this paper is threefold: (1) to describe a program for computing the conjugacy classes of $\Gamma(2),(2)$ to apply this to find the spectral variance of the Laplacian for character varieties, and (3) to study the remainders for the prime geodesic asymptotic formulas (see [3])

$$
\theta(x)=\sum \ln (N(\gamma)) \text { and } \pi(x)=\sum 1,
$$

where the sum is over the primitive conjugacy classes of norm $N(\gamma) \leq x$. The numerical data indicate that the Berry conjecture [2] for the spectral rigidity of strongly chaotic systems does not hold in this situation, even when the arithmetical symmetry is broken. Writing the remainder as $|\theta(x)-x|=x^{\beta}$, we find that the largest value of $\beta$ is $2 / 3$. This is somewhat of a surprise since the expected value was $1 / 2$. The remainder data turns out to have a negative bias; this is explained by the fact that the normalized remainders $(\theta(x)-x) / x^{1 / 2}$ and

Received by the editor September 14, 1993.

1991 Mathematics Subject Classification. Primary 11-04, 11Y70, 81Q50.

Work on this paper was supported in part by NSF Grant DMS-9303225. 


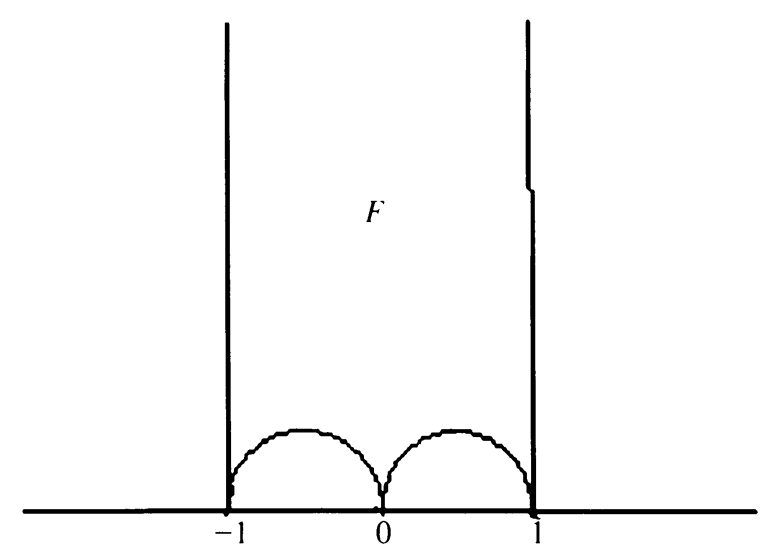

FIGURE 1

$(\pi(x)-\operatorname{li}(x)) \ln (x) / x^{1 / 2}$ ultimately have a negative mean, which, incidentally, implies an omega result for the remainders.

The program makes use of the fact that there is a one-to-one correspondence between the primitive conjugacy classes and the directed closed geodesics for a discrete subgroup of $\operatorname{PSL}(2, R)$. So to study a particular (not necessarily primitive) conjugacy class of trace greater than 2, we find a (hyperbolic) matrix $\alpha$ in this class whose directed axis intersects a fixed (convex) fundamental domain $F$; the segment of the axis in $F$ will be part of the directed closed geodesic corresponding to the conjugacy class. This axis leaves $F$ at two points going into two of the adjoining fundamental domains. Corresponding to each such side there is a generator of the group, taking $F$ into an abutting domain. If we conjugate $\alpha$ with respect to this generator we see that the axis of the conjugated matrix will again intersect $F$ and be a part of the original closed geodesic in $F$. In the next step there is only one possible exit from $F$ which does not backtrack (i.e., we eliminate from consideration the inverse of the conjugation used in the previous step). Continuing in this way, we eventually return to the original piece of the geodesic in $F$; that is, we get back to the matrix $\alpha$. The set of matrices encountered consists of all the matrices in the conjugacy class whose axes intersect $F$; we call such a set a chain. Each matrix in a chain appears in this set only once. The chain procedure in the program finds all chains of a given trace. This idea was previously used to compute the conjugacy classes for the "octagonal group" by Aurich et al. [1].

We shall study the subgroup

$$
\Gamma(2)=\{(a b, c d) \in \operatorname{PSL}(2, R) \text { congruent to } I \bmod (2)\},
$$

which, together with an associated character $\chi$, can be used to define the automorphic functions

$$
u(\gamma z)=\chi(\gamma) u(z), \quad \gamma \in \Gamma(2) .
$$

For $F$ we choose the fundamental domain depicted in Fig. 1. The program computes the number of primitive chains whose elements have a given trace (tr) as well as the value of $\chi$ on each such chain (a character does not change its value under conjugation).

Luo and Sarnak [5] have shown for cocompact arithmetic subgroups that a lower bound for the spectral variance of the twisted Laplacian can be obtained 
from the rate of growth $r$ of the series

$$
x[n]=\sum \ln ^{2}(\operatorname{tr}) \mu(\operatorname{tr})^{2},
$$

summed over all $\operatorname{tr} \leq 4 n+2$; here,

$$
\mu(\operatorname{tr})=\sum \chi(\text { chain })
$$

summed over all primitive chains of trace $\operatorname{tr}$. In the Luo-Sarnak development the spectral variance is bounded below by $L^{\alpha}$ in the universal range, where $\alpha=$ $r-2$. According to the Berry conjectures, $\alpha=1$ for integrable Hamiltonians, whereas for systems with chaotic dynamics the growth is logarithmic, in which case $\alpha$ will be zero.

We have taken over this measure of spectral rigidity for $\{\Gamma(2), \chi\}$ even though $F$ is not compact. However, the Luo-Sarnak development also holds for noncompact cofinite arithmetic groups if the $\chi$-twisted spectrum of the Laplacian is a pure point spectrum as it is for the generic $\chi$ in our case (see $\S 3$ for further details). It should be noted that the original notion of spectral rigidity may not be applicable for general nonarithmetic noncocompact subgroups in view of the strong possibility that the Laplacian for such systems may not have any (nonexceptional) point spectrum (see [8] and [11]).

As can be seen from Figures 4, 5, and 6, we found that $\alpha>0$ for all of the sampled values of $\chi$. These results conform with previous investigations. Numerical data obtained by Schmit [9] for the Dirichlet problem on the fundamental domain of a cocompact subgroup indicate that the spectral rigidity in this case is the same as that of integrable systems (i.e., $\alpha=1$ ). Luo and Sarnak [5] proved for twisted arithmetic cocompact subgroups that

$$
\alpha \geq 2\left(1-4 \lambda_{0}(\chi)\right)^{1 / 2}-1,
$$

where $\lambda_{0}(\chi)$ is the smallest eigenvalue of the twisted Laplacian.

Table 1 contains a list of the number of conjugacy classes (in parentheses) for each trace number $n \leq 1000$ (trace $=4 n+2$ ). In Fig. 7 we present a histogram of the remainder powers $\beta(x)$ (see above) for all of the trace numbers between 800 and 1200 . It will be noticed that most of the remainders are negative (i.e., $\theta(x)<x)$ and that the $\beta$ 's bunch up around the upper bound of $2 / 3$.

Section 4 contains a proof for all cofinite $\Gamma$ in $\operatorname{PSL}(2, R)$ that the mean normalized remainder (MNR) for $\theta(x)$ (Theorem 4.1) and $\pi(x)$ (Theorem 4.3) approaches a limit, which in the case of $\Gamma(2)$ is -7 . This explains the negative bias. The computed MNR for $\theta$ turns out to be slowly decreasing from -6.70 at $n=100$ to -6.90 at $n=1000$. On the other hand, the MNR data for $\pi$ are increasing from -8.23 at $n=100$ to -7.88 at $n=1000$ (see Table 2). These results suggest that the previous computations yield good approximations to the true asymptotic behavior of these various quantities.

\section{The CONJUGACY ClASSES OF $\Gamma(2)$}

There are several symmetries of $\Gamma(2)$ which can be used to shorten the program. It is easy to see that this group is closed under inverse, transposition and sign change of the off diagonal elements of a matrix (scod). We take these symmetries up in turn: 
(i) Inverse: A hyperbolic matrix in $\operatorname{PSL}(2, R)$ and its inverse share the same axis but the two axes are oppositely directed. Likewise, the set of inverses of the matrices in a chain is again a chain, and the corresponding closed geodesics are the same but oppositely directed. This means that the two chains are always distinct, and in our program they are treated separately.

(ii) Transposition: Corresponding to the four sides of $F$ are the four generators

$$
\begin{aligned}
& A=\left(\begin{array}{llll}
1 & 2,0 & 0
\end{array}\right), \quad A^{-1}=\left(\begin{array}{lll}
1 & -2,0 & 1
\end{array}\right),
\end{aligned}
$$

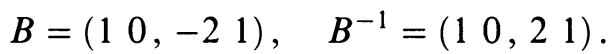

A conjugation involving any one of these matrices becomes, under transposition, another conjugation with $A \leftrightarrow B, A^{-1} \leftrightarrow B^{-1}$. If the transpose of one matrix in the chain is in the chain, then the transpose of all matrices in the chain are in the chain. Thus, the chain is either distinct from its transpose or self-transposed.

(iii) Scod: The conjugate of the scod of a given matrix by one of the four generators is the scod of the conjugate of the given matrix under the inverse of the same generator; i.e., $A \leftrightarrow A^{-1}, B \leftrightarrow B^{-1}$. This is evident from the following conjugations on changing the signs of $b, c$, and $j$ simultaneously:

$$
\begin{aligned}
& A^{j}(a b, c d) A^{-j}=\left(\begin{array}{lll}
a+2 j c & b+2 j d-2 j a-4 j^{2} c, c & d-2 j c
\end{array}\right), \\
& B^{j}(a b, c d) B^{-j}=\left(\begin{array}{lll}
a+2 j b & b, c-2 j a+2 j d-4 j^{2} b & d-2 j b
\end{array}\right) .
\end{aligned}
$$

We conclude from this that if a given chain is, say, self-transposed, then the scod and the scod of its transpose will also be self-transposed. Hence if a chain includes neither a transpose nor a scod of any particular matrix in the chain, then its transpose, its scod and the scod of its transpose will appear in distinct chains. If the transpose (or the scod (but not both)) of any particular matrix in the chain appears in the chain, then only the scod (or the transpose) chain will be distinct. Finally, if both the transpose and the scod of some matrix in the chain belong to the chain, then the chain will be self-transposed and selfscod. This permits us to limit our computation to only one of the four (two or one) possible chains connected by transposition and scod. Moreover, all of these considerations hold for the inverse chains for which the character $\chi$ is the complex conjugate of the $\chi$ for the original chain. Thus, the imaginary part of the $\chi$ contribution is cancelled out, and we need only retain the real part.

Proposition 1. The traces of hyperbolic matrices in $\Gamma(2)$ are of the form $\mathrm{tr}=$ $4 n+2$ for $n$ a positive integer.

Remark. Because we are treating $\Gamma(2)$ in $\operatorname{PSL}(2, R)$, we can limit ourselves to positive traces. In what follows we shall call $n$ the trace number.

Proof of Proposition 1. Since $a$ and $d$ are odd we can write $a=2 j+1$ and $d=2 k+1$. Then

$$
a d-1=4 j k+2(j+k)=b c .
$$

Since the right-hand side is divisible by $4,(j+k)$ has to he even, and since $\operatorname{tr}=a+d=2(j+k)+2$, we see that $\operatorname{tr}$ is of the form $4 n+2$. If the matrix is hyperbolic, then $\operatorname{tr}>2$, and hence $n>0$.

It is easy to see that each conjugacy class with $\operatorname{tr}>2$ contains matrices whose axes intersect $F$. Since the union of the $\{\gamma F, \gamma \in \Gamma(2)\}$ fills out the hyperbolic 
plane, the axis of any matrix $\alpha$ in this class will intersect some $\gamma^{-1} F$, and the axis of $\beta=\gamma \alpha \gamma^{-1}$ will intersect $F$. For the fundamental domain $F$ with cusps at $-1,0,1$ and $\infty$ this can happen if and only if the fixed points of $\beta$ straddle the point $-1,0$, or 1 on the real axis. Note that the points $-1,0,1$, and $\infty$ do not belong to $F$.

Lemma 2. Suppose $\alpha=(a b, c d)$ is hyperbolic. The fuxed points of $\alpha$ will straddle 0 if and only if $0<a<\operatorname{tr}, 1$ if and only if $0<a-c<\operatorname{tr}$, and -1 if and only if $0<a+c<\mathrm{tr}$.

Proof. The fixed points of $\alpha$ are

$$
x_{ \pm}=\left(2 a-\operatorname{tr} \pm\left(\operatorname{tr}^{2}-4\right)^{1 / 2}\right) / 2 c=(2 a-\operatorname{tr} \pm \operatorname{tr}) / 2 c \mp \delta / c,
$$

where, since $\operatorname{tr} \geq 6$,

$$
0<\delta=\frac{\operatorname{tr}}{2}\left(1-\left(1-4 / \operatorname{tr}^{2}\right)^{1 / 2}\right)<2 / \operatorname{tr}<1 / 2
$$

If $c>0$, then $x_{-}<1<x_{+}$if and only if

$$
a-\operatorname{tr}+\delta<c<a-\delta .
$$

Since $a$ and $c$ are integers, we see that this condition is the same as $0<a-c<$ $\operatorname{tr}$. On the other hand, if $c<0$, then $x_{+}<1<x_{-}$if and only if

$$
a-\delta>c>a-\operatorname{tr}+\delta .
$$

This is exactly the same condition as before. This proves the second assertion of the lemma, and the others are proved in a similar fashion.

For a hyperbolic matrix, if $0<a<\operatorname{tr}$, then $a+d>2, a d=a(\operatorname{tr}-a)>1$ and $b c=a d-1>0$, so that $b$ and $c$ have the same sign. Moreover, neither $b$ nor $c$ can vanish, since otherwise $a d=1$.

Definition. A matrix whose axis intersects $F$ is called an admissible matrix and a zero-admissible matrix if its axis straddles 0 (i.e., if $0<a<\operatorname{tr}$ ); if in addition $0<b \leq c$, it is called a list matrix.

It is easy to see that there are only a finite number of list matrices. In the first place, $a=2 k+1$ can take on only $\operatorname{tr} / 2$ values. For each such choice of $k$,

$$
b c=a d-1=(2 k+1)(4 n+2-2 k-1)-1=4\left(2 k n+n-k^{2}\right) .
$$

There are at most $\left(2 k n+n-k^{2}\right)^{1 / 2}$ choices for $b / 2,0<b \leq c$, and once $b$ is chosen, $c$ is uniquely determined by the relation (2.5). The list procedure in the program carries out the calculation which we have just outlined for $0<b \leq c$. The transpose and the scod of the above matrices also have $0<a<\operatorname{tr}$, so that the number of zero-admissible matrices is less than or equal to four times the number of list matrices computed above. In the program the arrays $a, b, c$ enumerate the elements in the list matrices. The $f$ array is a flag, which at any given point in the program is 1 if the corresponding list matrix (or its transpose or scod) has been used, and is otherwise 0 .

There is a simple correspondence between the admissible matrices for which $0<a<\operatorname{tr}$ and those for which $0<a-c<\operatorname{tr}$ or $0<a+c<\operatorname{tr}$. In fact, (2.6)

$$
(1 \mp 1,01)(a b, c d)(1 \pm 1,01)=\left(a \mp c b^{\prime}, c d \pm c\right), \quad b^{\prime}= \pm(a-d)+b-c \text {. }
$$




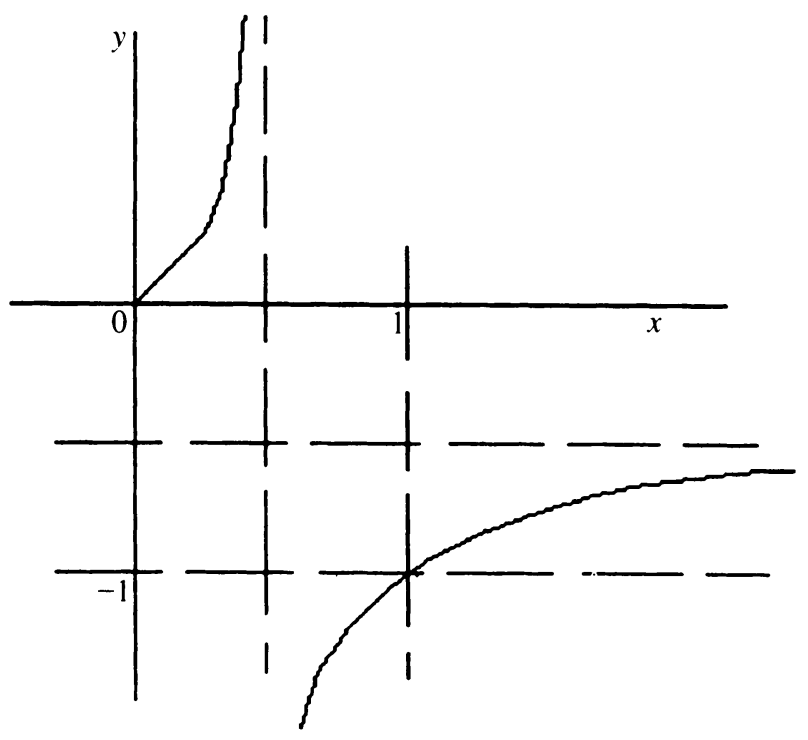

FIGURE 2. $y=-x /(2 x-1)$

Thus, suppose $0<a-c<\operatorname{tr}$ and, say, $c>0$ (otherwise write this as $0<a+(-c)<\operatorname{tr}$ with $-c>0$ ). Then the matrix on the right (using the upper sign) is a zero-admissible matrix with the same trace. This shows that the number of admissible matrices is at most four times the number of zeroadmissible matrices. We conclude from this that the number of steps in any chain procedure is finite and that each closed geodesic has only a finite number of axis segments. The next lemma shows that when we exhaust the list matrices in the chain procedure (which also takes care of their transposes and scods), we will automatically exhaust all of the admissible matrices. Notice that the scod of the set of list matrices includes their inverses.

Lemma 3. Each chain contains at least one zero-admissible matrix.

Proof. It suffices to consider an admissible matrix $\gamma=(a b, c d)$ whose fixed points $x_{ \pm}$satisfy the conditions $0<x_{-}<1$ and $x_{+}>1$. Suppose we conjugate with respect to $B$. The fixed points of $\gamma^{\prime}=B \gamma B^{-1}$ will be $y_{ \pm}=$ $B x_{ \pm}: y=-x /(2 x-1)$; see Fig. 2. In any case, $0>y_{+}>-1$. If $0<x_{-}<1 / 2$, then $y_{-}>0$, and $\gamma^{\prime}$ will be a zero-admissible matrix, and the lemma is valid. If $x_{-}=1 / 2$, then $\left|y_{-}\right|=\infty$, and it follows from (2.3) that $c^{\prime}=0$, which is impossible for a hyperbolic matrix in $\Gamma(2)$. Finally, if $1>x_{-}>1 / 2$, then $y_{-}<-1$, and $\gamma^{\prime}$ is admissible and a member of the chain.

Next we conjugate $\gamma^{\prime}$ by $A$ to get $\gamma^{\prime \prime}=A B \gamma(A B)^{-1}$ whose fixed points are $z_{ \pm}=A B x_{ \pm}: z=(3 x-2) /(2 x-1)$; see Fig. 3 . In any case, $z_{+}>1$. If $1 / 2<x_{-}<2 / 3$, then $z_{-}<0$, and $\gamma^{\prime \prime}$ is a zero-admissible matrix, and again the lemma is valid. If $2 / 3 \leq x_{-}<1$, then $0 \leq z_{-}<1$, and $\gamma^{\prime \prime}$ is admissible with $z_{-}$and $z_{+}$straddling 1 as in the case of $\gamma$. We now show that as we iterate this process the $x_{-}$decreases, with the absolute value of the increment of decrease bounded from below. In fact,

$$
\delta=x-z=x-(3 x-2) /(2 x-1)=x(x-1)^{2} /(2 x-1) .
$$

As $x_{-}$approaclies $1 / 2$ from above, $\delta$ grows and $x_{-}$becomes less than $2 / 3$ 


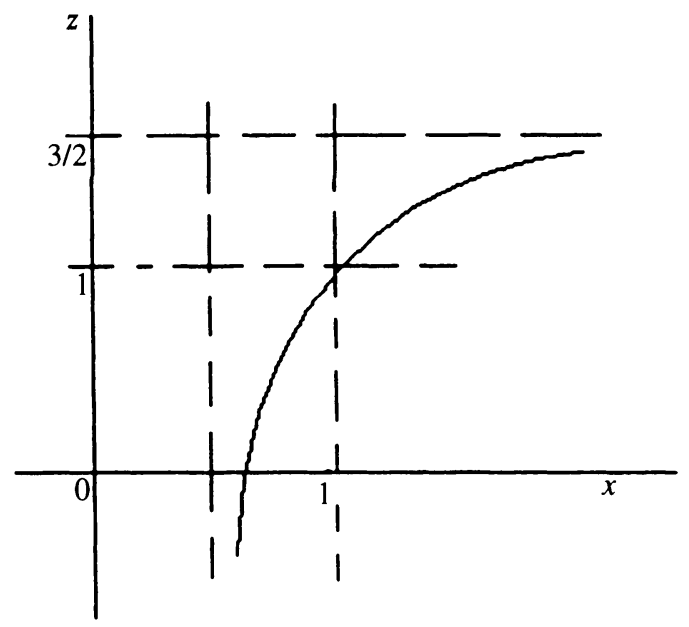

FiguRE 3. $z=(3 x-2) /(2 x-1)$

(and hence the corresponding $\gamma^{\prime \prime}$ is zero-admissible) after a finite number of steps. This concludes the proof of the lemma.

We are now in a position to explain the structure of the program. The chain procedure computes all of the chains of a given trace $=4 n+2$ modulo their transpositions and scods. It does so by systematically going through the list matrices (with $0<b \leq c$ ) and starting a new chain with the first such matrix which has not been in any previous chain; the latter is denoted by $a[i], b[i], c[i]$. This selection is performed, with the help of the flag array $f$, by the "repeat" statement at the end of the procedure. After setting the initial chain matrix $(a 1, b 1, c 1)$ equal to the so selected list matrix, the program goes on to the link procedure, which then repeatedly determines the next link in the chain. However, just before this, the program initializes $e, s$, and $t$. The conjugations defined by the four generators (2.1) are enumerated by $e$, and the $o 1$ array records the inverse of the previously used conjugation. The variable $s$ is a flag which is initially 0 but is set equal to one in the link procedure when the final link in the chain is attained (i.e., when the link calculation finally returns to its starting matrix). The value $s=1$ terminates the first "repeat" statement in the chain procedure. The variable $t$ is also a flag which is adjusted so that at the end of a chain computation it is equal to 0 if the chain is neither selftransposed nor self-scod, equal to 1 if it is self-transposed or self-scod (but not both), and equal to or greater than 2 if it is both self-transposed and self-scod. If $b[i]=c[i]$, then $t$ starts off as 1 , since the chain is then necessarily selftransposed. The chain procedure ends by summing up the $\chi$-values of each of the chains of trace number $n$.

The link procedure computes the next link in the chain. It starts by making sure that the inverse of the previously used conjugation is not used again. It then proceeds to calculate successively the other conjugations until it reaches an admissible matrix. At each step it sets the $a 1, b 1, c 1$ array parameter $u$ ahead by 1 . First it checks to see whether the condition $0<a<\operatorname{tr}$ is satisfied by $a 1[u]$. If so, it removes $(a=a 1[u], b=|b 1[u]|)$ or $(a=a 1[u], b=|c 1[u]|)$ from the available list matrices by changing the corresponding matrix $f$-value 
to 1 . Then it checks to see whether or not $(a 1[u], b 1[u], c 1[u])$ is equal to the starting matrix. If not, it initializes $e$ to 0 for the next link procedure and determines whether it is the transpose or scod of the starting matrix; in either case it increases $t$ by 1 . However, when $b[i]=c[i]$, the transpose option is not realized (the scod option remains), and in this case we have to rely on the initializing of $t$ in the chain procedure to advance $t$ by 1 . Finally, if $(a 1[u], b 1[u], c 1[u])$ is equal to the starting matrix, then $s$ is set equal to 1 to end the chain. If the condition $0<a<\operatorname{tr}$ is not met, the program checks to see whether the companion condition $0<a \pm c<\operatorname{tr}$ is met. If so, the matrix is admissible and can be added to the chain and $e$ is reset to 0 . If not, the matrix does not belong to the chain and we return (through chain) to link with $e$ increased by 1 (modulo 4 ) and the array parameter $u$ reset. This last option can be iterated at most three times since one of the conjugations has to result in an admissible matrix.

Back in the chain procedure the program combines the contributions of all the chains of trace number $n$ by summing the chain characters. The quantity $e 1$ is equal to one-half of the contribution of a given chain, its transpose, its scod, and the scod of its transpose. If $t=0$, then we combine the contributions of the computed chain with that of the transpose, the scod and the transposedscod to get $2 e 1$. If $t=1$, then the chain is either self-transposed or self-scod (but not both), and there is only the contribution of two chains to be considered, so $e 1$ is added. Finally, if $t \geq 2$, then the chain is both self-transpose ard selfscod, and there is only the contribution of this one chain to take care of, so $e 1 / 2$ is added.

So far we have neglected the evaluation of the character $\chi$. The program computes the sum (1.4) for all trace numbers $\leq \max$, and this for $d$ different characters parameterized by the $k, l, r$ arrays. To describe $\chi$, we make use of the fact that $\Gamma(2)$ is a free group generated by $A$ and $B$. Each element of the group can be uniquely expressed as

$$
\alpha=A^{p_{1}} B^{q_{1}} A^{p_{2}} B^{q_{2}} \cdots A^{p_{k}} B^{q_{k}} .
$$

The character is determined by two parameters $\xi, \eta, 0 \leq \xi, \eta \leq 1$ :

$$
\chi_{(\xi, \eta)}(A)=\exp (2 \pi i \xi), \quad \chi_{(\xi, \eta)}(B)=\exp (2 \pi i \eta)
$$

and

$$
\chi_{(\xi, \eta)}(\alpha)=\exp (2 \pi i(p \xi+q \eta)), \quad p=\sum p_{j}, q=\sum q_{j} .
$$

In the program the char procedure computes $p$ and $q$ for the initial list matrix, and these are used in the chain procedure to compute $e 1$. To understand how this is done, we need the following

Proposition 4. Suppose that $\alpha$ is the starting hyperbolic matrix for a chain, and $\gamma$ is the product of the conjugating matrices culminating with $\alpha=\gamma \alpha \gamma^{-1}$. If $\alpha$ is primitive, then $\gamma$ is equal to $\alpha$ or its inverse. If $\alpha=\alpha_{0}^{k}$ and $\alpha_{0}$ is primitive, then $\gamma=\alpha_{0}$ or its inverse.

Proof. Clearly, $\alpha$ and $\gamma$ commute, and this implies that they have the same fixed points. If $\alpha$ is primitive, this means that $\gamma=\alpha^{k}$ for some $k \in Z$. On the other hand, $\gamma$ has to be primitive since, if $k$ is not of absolute value 1 , the chain computation would have ended when the cumulative conjugation reached 
$\alpha$ (or its inverse if $k<0$ ). This proves the first assertion, and the second follows by a similar argument.

When the current conjugating matrix results in an admissible matrix, then it is fed into the char procedure as $o 1[u]+2(\bmod 4)$. If this is $A\left(\right.$ or $\left.A^{-1}\right)$, then 1 (or -1 ) is added to $p$, whereas if this is $B$ (or $B^{-1}$ ), then 1 (or -1 ) is added to $q$. In the computation of $e 1$ we must keep in mind that the contribution of the transpose interchanges $p$ and $q$ while that of the scod changes the signs of $p$ and $q$.

Finally we note that in the main part of the program we eliminate all nonprimitive conjugacy classes by keeping track of when such classes can occur. Suppose the starting matrix $\alpha$ has eigenvalues $\lambda$ and $1 / \lambda$; then $\operatorname{tr}(\alpha)=\lambda+1 / \lambda$ and $\operatorname{tr}\left(\alpha^{k}\right)=\lambda^{k}+1 / \lambda^{k}$. A straightforward calculation shows that in terms of the trace numbers $n_{k}$ we obtain for the trace of $\alpha^{k}$

$$
\begin{aligned}
& n_{2}=4\left(n_{1}^{2}+n_{1}\right), \\
& n_{3}=16 n_{1}^{3}+24 n_{1}^{2}+9 n_{1}, \\
& n_{4}=64 n_{1}^{4}+128 n_{1}^{3}+80 n_{1}^{2}+16 n_{1} .
\end{aligned}
$$

If we limit ourselves to the first 1000 trace numbers, then $n_{2}$ occurs only for $1 \leq n_{1} \leq 15, n_{3}$ occurs only for $1 \leq n_{1} \leq 3$, and $n_{4}$ only for $n_{1}=1$.

One check on the program is to see whether its results remain the same if we conjugate by elements of the modular group. As shown in [7], this amounts to transforming the $\chi$-parameters $(\xi, \eta)$ by the matrices $(10,01),(01,10)$, $(10,-1-1),(-1-1,10),(01,-1-1)$, and $(-1-1,01)$. The program did pass this test.

\section{NUMERICAL RESULTS ON SPECTRAL RIGIDITY}

Next we discuss the output of the program. Our original aim was to find the rate of growth of the series $x[n]$ described in (1.4). To this end, we determined the logarithm of $x[n]$ as a function of the logarithm of the trace $(4 n+2)$ of the $n$th trace number. Since the series grows somewhat irregularly, we computed the slope of the best mean square linear fit over sets of 150 successive trace numbers, starting at the trace number 751-900 and ending at 851-1000 in increments of 15 . For each such run the slope is given by

$$
\text { slope }=\left(b \sum \ln (x[j])-a \sum \log (\operatorname{tr}(j)) \ln (x[j])\right) /\left(b^{2}-a c\right),
$$

where

$$
a=\sum 1, \quad b=\sum \log (\operatorname{tr}(j)) \quad \text { and } \quad c=\sum\left(\log (\operatorname{tr}(j))^{2} ;\right.
$$

here all the summations are over the particular set. We recorded the average $r$ of these 11 slopes and the square root of the variance; the latter we took as the probable error. Because of obvious symmetries, it sufficed to consider only character parameters in the triangle $0 \leq \eta \leq \xi \leq 1 / 2$.

Figures 4, 5, and 6 depict the results for runs of 1000 trace numbers, entailing $1,031,772$ primitive conjugacy classes. Each figure consists of computed data (i.e., the mean slope together with the probable error) for each run and a smooth 


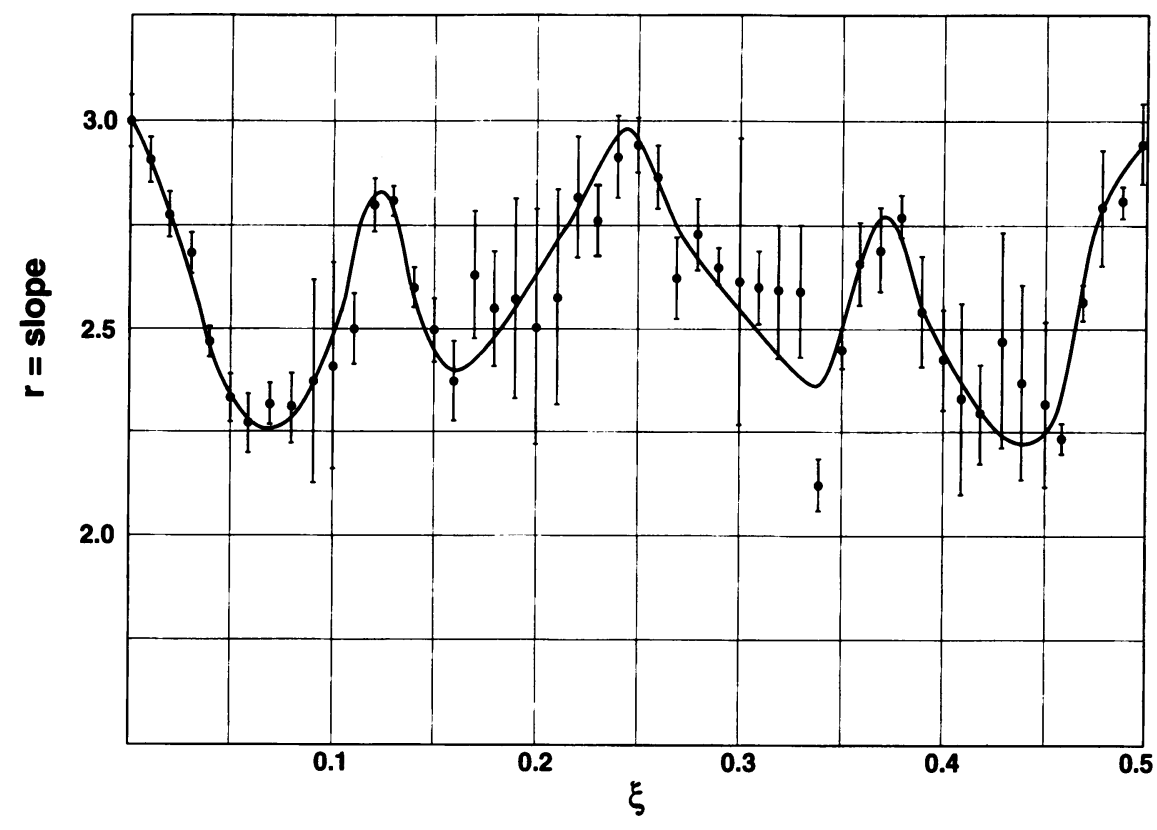

FIGURE 4. $\xi=i / 100, \eta=0$

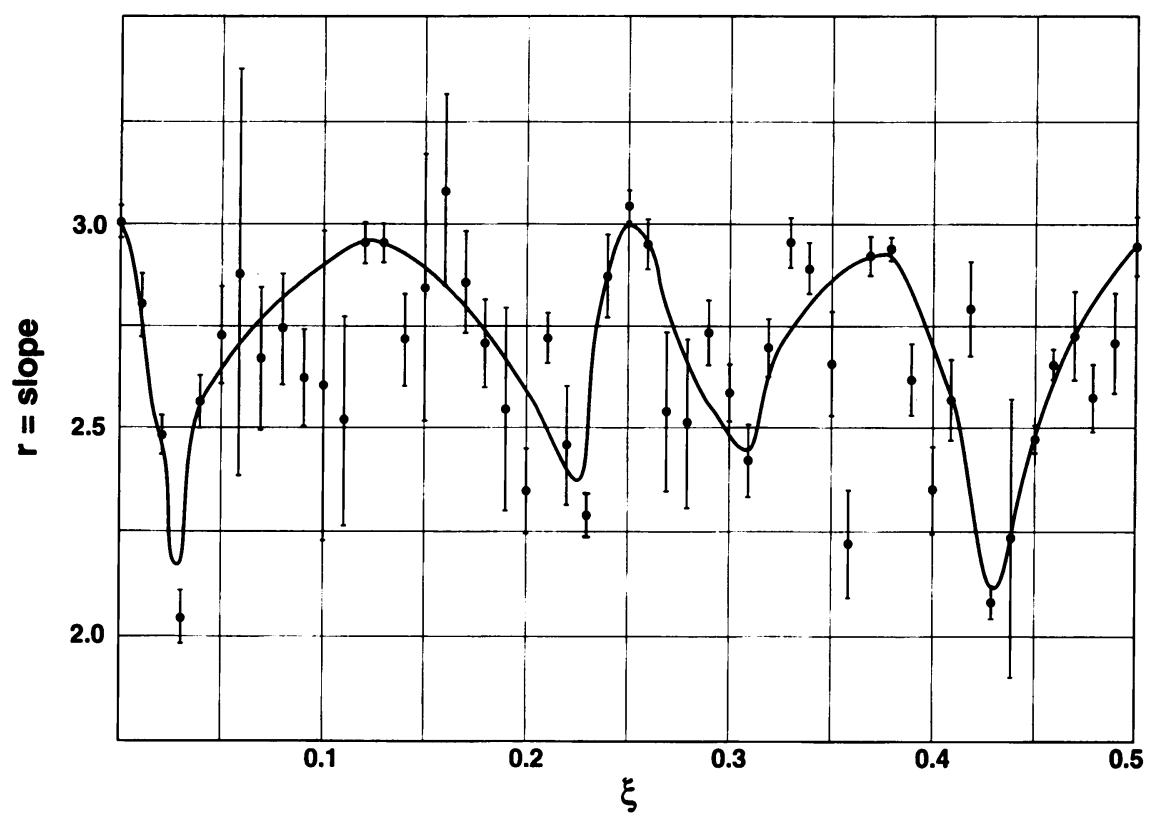

FIGURE 5. $\xi=i / 100, \eta=i / 100$

curve which roughly fits the data. The character parameters for Fig. 4 are $\xi=i / 100, \eta=0$; those for Fig. 5 are $\xi=i / 100, \eta=i / 100$, and those for Fig. 6 are $\xi=i / 100, \eta=i / 200(0 \leq i \leq 50)$. 


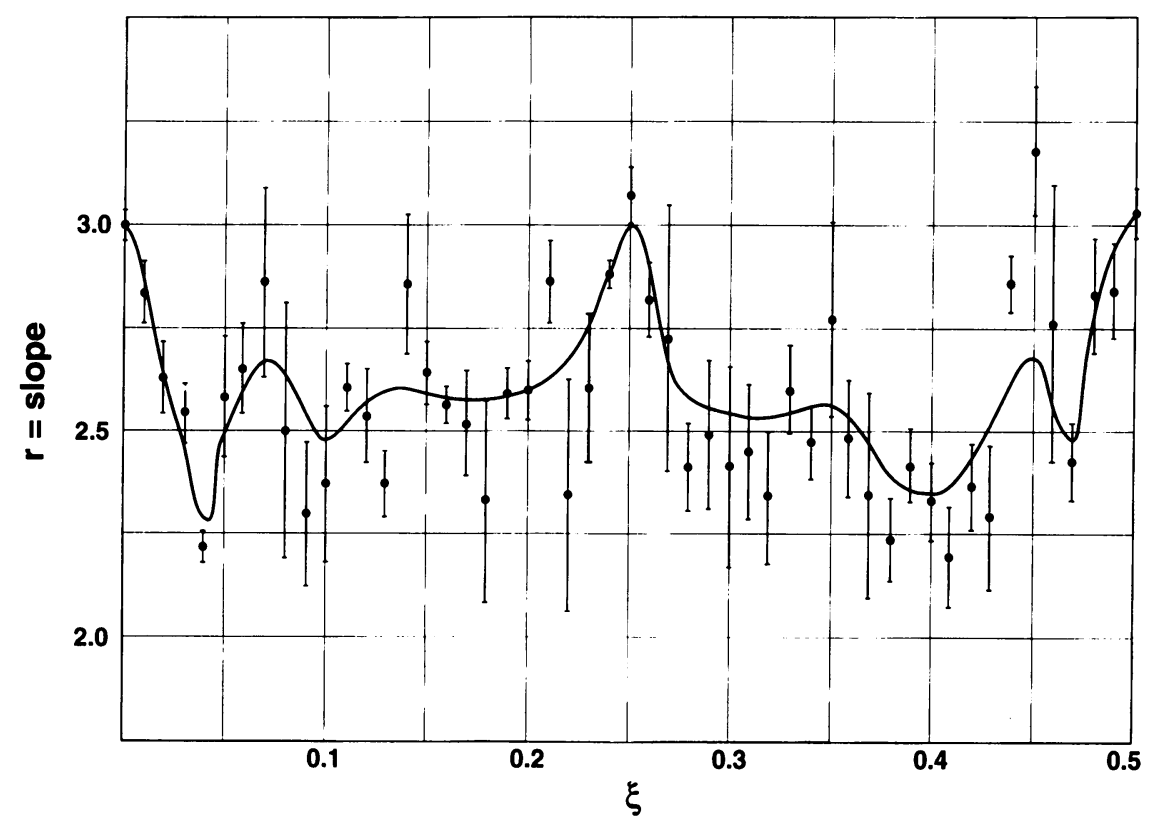

FIGURE 6. $\xi=i / 100, \eta=i / 200$

The slope was found to be approximately equal to 3 at parameter values $(i / 8, j / 8)$ for integer values of $i, j$ less than or equal to 7 . These points are closely related to congruent subgroups (see [7]). For all of the sampled parameters the slope was greater than 2. As explained in the introduction, such slopes are not what is expected for these strongly chaotic systems. All of the curves display a semblance of symmetry about the line $\xi=1 / 2$. A copy of the program, written in Pascal, can be found in the Supplement section at the end of this issue.

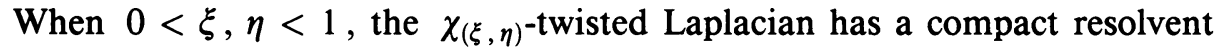
and hence the Luo-Sarnak theory is valid. This means that for such $\chi$ the growth of the spectral rigidity is at least $L^{\alpha}$, where $\alpha=r-2$. In order to say something about $\chi$ 's for which $\eta=0$ (or $\xi=0$ ), we would have to assume that the spectral rigidity is continuous in $\chi$, which is not known. However, the partial sums (1.4) are obviously continuous in $\chi$, and hence the data in Fig. 4 approximate the values for the slope when $(\xi, \eta)$ (with $0<\xi, \eta<1$ ) is close to $(\xi, 0)$.

A slight modification of the last section of the program allowed us to compute the number of conjugacy classes (in parentheses) for each trace number $n \leq$ 1000 (see Table 1) and the remainder power $\beta(x)$ for the function $|\theta(x)-x|=$ $x^{\beta}$ (see (1.1)). A histogram of these $\beta$ 's for trace numbers $n$ between 800 and 1200 is depicted in Fig. 7. This involves $1,450,632$ conjugacy classes. As can be seen, the maximum $\beta$ is $2 / 3$. By assuming the Lindelöf hypothesis for certain $L$-functions, Iwaniec [4] can prove an upper bound of $2 / 3$. Without this assumption, the best upper bound (also due to Iwaniec [4]) is $35 / 48$. 
TABLE 1. Trace number (number of conjugacy classes)

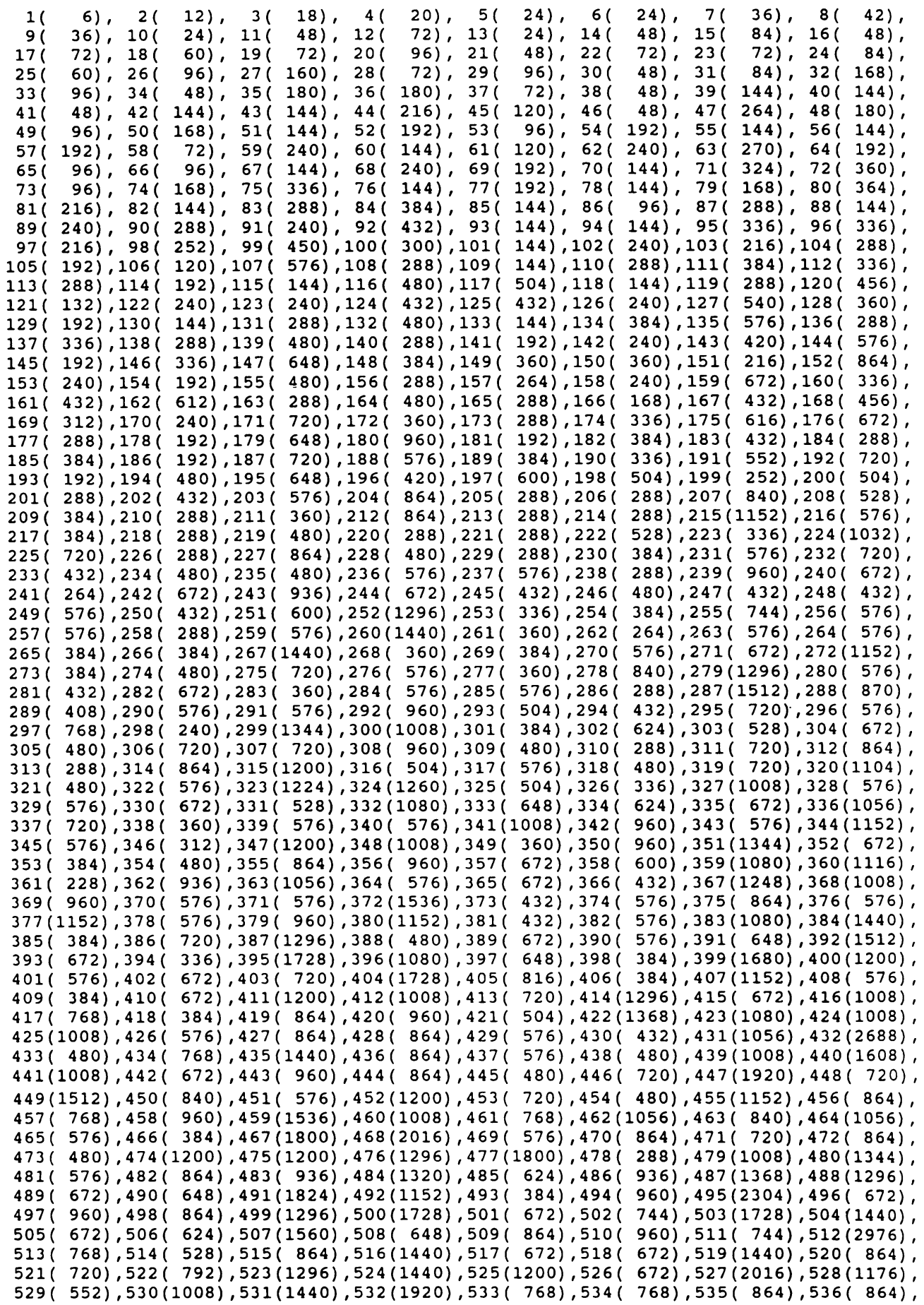




\section{TABLE 1 (continued)}

$537(960), 538(648), 539(2240), 540(1728), 541(360), 542(912), 543(1008), 544(1344)$, $545(1152), 546(864), 547(864), 548(1200), 549(1512), 550(672), 551(1152), 552(1440)$, $553(672), 554(960), 555(1728), 556(648), 557(1368), 558(720), 559(1056), 560(1344)$, $561(768), 562(864), 563(720), 564(1920), 565(912), 566(1224), 567(2592), 568(1008)$, $569(672), 570(576), 571(720), 572(2304), 573(672), 574(672), 575(3150), 576(2016)$, $577(816), 578(816), 579(1728), 580(960), 581(672), 582(1152), 583(720), 584(1728)$, $585(960), 586(840), 587(2352), 588(1296), 589(768), 590(768), 591(1008), 592(1056)$, $593(1152), 594(1536), 595(864), 596(1440), 597(1056), 598(480), 599(1080), 600(2160)$, $601(816), 602(1440), 603(2520), 604(936), 605(1056), 606(624), 607(1512), 608(1344)$ $609(768), 610(960), 611(1728), 612(2400), 613(504), 614(1152), 615(1152), 616(864)$, $617(1584), 618(720), 619(1344), 620(2304), 621(1152), 622(864), 623(1920), 624(2064)$, $625(600), 626(576), 627(2016), 628(1152), 629(1440), 630(1584), 631(1296), 632(1296)$, $633(960), 634(576), 635(1920), 636(1008), 637(864), 638(1296), 639(1800), 640(1440)$, $641(672), 642(1824), 643(1008), 644(1920), 645(960), 646(576), 647(2268), 648(1224)$, $649(960), 650(960), 651(1536), 652(2088), 653(624), 654(864), 655(1344), 656(2880)$, $657(1728), 658(576), 659(1728), 660(1920), 661(408), 662(1056), 663(1440), 664(1440)$, $665(1152), 666(1200), 667(1440), 668(1008), 669(1152), 670(1056), 671(1344), 672(2016)$, $673(576), 674(1344), 675(2178), 676(780), 677(1872), 678(864), 679(1152), 680(1440)$, $681(960), 682(816), 683(1680), 684(2160), 685(1152), 686(1152), 687(2112), 688(1344)$, $689(960), 690(960), 691(1080), 692(3744), 693(960), 694(720), 695(1152), 696(1152)$, $697(768), 698(1200), 699(1680), 700(1512), 701(1344), 702(1536), 703(1104), 704(1440)$, $705(1344), 706(528), 707(2592), 708(2400), 709(720), 710(960), 711(2160), 712(1728)$, $713(768), 714(1152), 715(2112), 716(1008), 717(1056), 718(528), 719(3528), 720(2640)$, $721(912), 722(2016), 723(864), 724(1920), 725(1320), 726(936), 727(1584), 728(1896)$ $729(1944), 730(672), 731(1440), 732(2016), 733(840), 734(1296), 735(2352), 736(1680)$, $737(1440), 738(1008), 739(1152), 740(1920), 741(960), 742(864), 743(1584), 744(1728)$ $745(576), 746(1368), 747(4320), 748(1152), 749(1152), 750(1440), 751(1248), 752(1680)$, $753(768), 754(1056), 755(2304), 756(2304), 757(1080), 758(864), 759(1728), 760(1152)$ $761(1008), 762(1632), 763(1440), 764(2160), 765(1584), 766(720), 767(2256), 768(1488)$, $769(672), 770(1152), 771(1440), 772(1440), 773(1800), 774(1800), 775(1440), 776(2016)$, $777(1152), 778(720), 779(1920), 780(1728), 781(576), 782(1920), 783(3024), 784(2016)$, $785(1632), 786(672), 787(1800), 788(2016), 789(1152), 790(864), 791(1440), 792(4320)$ $793(576), 794(864), 795(1440), 796(1512), 797(1344), 798(960), 799(2352), 800(3528)$, $801(960), 802(1056), 803(1728), 804(1920), 805(960), 806(1248), 807(2304), 808(720)$, $809(1632), 810(2376), 811(1344), 812(1728), 813(1248), 814(768), 815(2112), 816(1344)$, $817(1536), 818(1200), 819(2592), 820(1536), 821(1008), 822(1200), 823(864), 824(3024)$ $825(1344), 826(816), 827(3960), 828(1800), 829(960), 830(960), 831(2880), 832(2576)$, $833(864), 834(1152), 835(1152), 836(1920), 837(1344), 838(1128), 839(2016), 840(2064)$ $841(1044), 842(1296), 843(1824), 844(1080), 845(1800), 846(1584), 847(2184), 848(2640)$, $849(1440), 850(1200), 851(1440), 852(3840), 853(672), 854(2304), 855(2160), 856(864)$, $857(1248), 858(960), 859(1680), 860(1728), 861(1440), 862(1152), 863(2016), 864(2688)$, $865(960), 866(912), 867(2736), 868(1440), 869(1536), 870(960), 871(864), 872(2880)$, $873(2160), 874(1152), 875(3456), 876(2592), 877(936), 878(912), 879(1920), 880(2016)$ $881(1296), 882(2100), 883(1152), 884(2688), 885(864), 886(792), 887(3600), 888(1440)$, $889(960), 890(2592), 891(2040), 892(1296), 893(960), 894(1728), 895(2160), 896(1440)$, $897(2112), 898(816), 899(2700), 900(3600), 901(864), 902(1536), 903(1440), 904(1440)$ $905(1248), 906(1296), 907(2160), 908(2376), 909(1920), 910(960), 911(2352), 912(2688)$,, $913(768), 914(864), 915(1728), 916(2400), 917(3072), 918(1728), 919(1584), 920(1728)$ $921(864), 922(1536), 923(1920), 924(2016), 925(1176), 926(1200), 927(3528), 928(2016)$ $929(1632), 930(2016), 931(1296), 932(3120), 933(864), 934(864), 935(2592), 936(2160)$, $937(1008), 938(1248), 939(3648), 940(1008), 941(1296), 942(1728), 943(2112), 944(2688)$ $945(2304), 946(864), 947(2016), 948(1536), 949(1008), 950(2016), 951(1440), 952(2592)$, $953(1680), 954(1584), 955(1440), 956(1728), 957(1920), 958(768), 959(2304), 960(2.28)$, $961(744), 962(2736), 963(2160), 964(1920), 965(1152), 966(864), 967(2772), 968(1872)$, $969(1536), 970(816), 971(4368), 972(3744), 973(720), 974(2640), 975(3360), 976(1056)$ $977(1152), 978(1152), 979(2016), 980(4320), 981(1944), 982(1320), 983(1584), 984(1728)$ $985(1536), 986(1152), 987(2880), 988(1440), 989(1440), 990(1680), 991(1344), 992(3696)$ $993(1440), 994(1056), 995(2016), 996(1920), 997(1608), 998(960), 999(3456), 1000(1728)$

sum of conjugacy classes $=1031772$ 


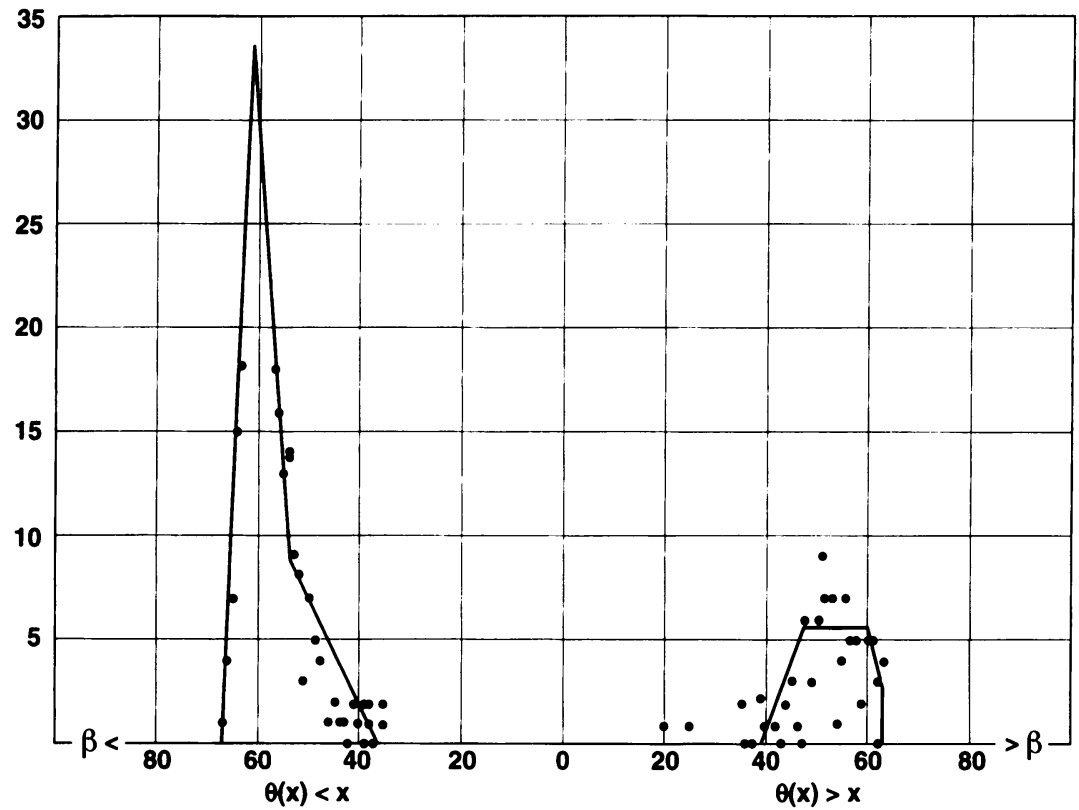

Figure 7. Histogram. Remainder $=|\theta(x)-x|=x^{\beta} ; 800 \leq$ trace number $\leq 1200$

There are examples in number theory where the true asymptotic nature of a series does not reveal itself until very far out in the series, and it could be that this is the case here. However, as we show in the next section, the negative bias of the remainders $(\theta(x)-x)$ and $(\pi(x)-\operatorname{li}(x))$ holds up in the limit. In fact, the limit of the mean normalized remainder (MNR) is -7 in both cases. For $\theta$ the data for trace numbers $n \leq 1000$ (starting at $n=51$ ) produced an MNR of -6.90 , and the MNR for $\pi$ was -7.88 at $n=1000$ (see Table 2). Both results are reasonably close to the asymptotic limit. The MNR formula is for an integrated mean, so we evaluated the $x$ in $(\theta(x)-x)$ at the midpoint between $4 n+2$ and $4 n+6$ for each trace number. There are two reasons why the MNR values for $\pi$ are less reliable than those for $\theta:(1)$ we compensated for the integral mean by taking the values of $\operatorname{li}(x)$ for the $n$th trace number to be the average of its values at the traces $4 n+2.3+i, i=0,1,2,3$; using 2.3 instead of 2.5 allows for the decreasing slope of $\operatorname{li}(x)-a$ change of 0.1 in the trace results in a change of -0.2 in the MNR and (2) the MNR was computed using an averaging function with an abrupt discontinuity at $s=1$, whereas the proofs of Theorems 4.1 and 4.3 require a smooth averaging function, smoother for $\pi$ than for $\theta$. Using our formula (4.26), V. Golovchansky and M. Smotrov have obtained similar results for the MNR of $\pi$ for $\Gamma_{0}(N)$ with several $N$ 's (personal communication).

Finally, we mention that for character parameters $\xi=1 / 8$ and $3 / 8, \eta=0$, and for $n \leq 1000$ we found that $\mu(n)=0($ see $(1.5))$ for $n=1,2 \bmod 4$ except for $n=1$ and 49 . 
TABle 2. The MNR (a) for $\theta$ and (b) for $\pi$. Column 1: trace number; 2: number of conjugacy classes; 3 : $\theta(x)$ for $(a)$ and $\pi(x)$ for (b); 4: MNR

$\begin{array}{rr}100 & 300 \\ 150 & 360 \\ 200 & 504 \\ 250 & 432 \\ 300 & 1008 \\ 350 & 960 \\ 400 & 1200 \\ 450 & 840 \\ 500 & 1728 \\ 550 & 672 \\ 600 & 2160 \\ 650 & 960 \\ 700 & 1512 \\ 750 & 1440 \\ 800 & 3528 \\ 850 & 1200 \\ 900 & 3600 \\ 950 & 2016 \\ 1000 & 1728\end{array}$

$\begin{array}{rr}163229 & -6.70 \\ 361686 & -6.86 \\ 641659 & -6.74 \\ 996198 & -6.99 \\ 1452763 & -6.65 \\ 1959925 & -6.69 \\ 2571805 & -6.91 \\ 3249160 & -6.98 \\ 4016388 & -7.10 \\ 4840320 & -6.71 \\ 5758380 & -6.85 \\ 6758825 & -6.75 \\ 7825128 & -6.83 \\ 9021235 & -6.86 \\ 10271902 & -6.89 \\ 11559333 & -6.89 \\ 12995242 & -6.89 \\ 14432211 & -6.89 \\ 16010282 & -6.90\end{array}$

(a)

$\begin{array}{rrrr}100 & 300 & 14938 & -8.23 \\ 150 & 360 & 30862 & -8.29 \\ 200 & 504 & 52202 & -8.10 \\ 250 & 432 & 78242 & -8.29 \\ 300 & 1008 & 110816 & -7.91 \\ 350 & 960 & 146156 & -7.91 \\ 400 & 1200 & 187964 & -8.09 \\ 450 & 840 & 233468 & -8.13 \\ 500 & 1728 & 284252 & -8.24 \\ 550 & 672 & 338092 & -7.82 \\ 600 & 2160 & 397378 & -7.94 \\ 650 & 960 & 461302 & -7.82 \\ 700 & 1512 & 528772 & -7.88 \\ 750 & 1440 & 603772 & -7.89 \\ 800 & 3528 & 681532 & -7.91 \\ 850 & 1200 & 760968 & -7.90 \\ 900 & 3600 & 848928 & -7.89 \\ 950 & 2016 & 936360 & -7.88 \\ 1000 & 1728 & 1031772 & -7.88\end{array}$

(b)

\section{THE MEAN NORMALIZED REMAINDER}

We see from Fig. 7 that the remainder $(\theta(x)-x)$ exhibits a negative bias. In this section we explain this bias by showing that the mean normalized remainder,

$$
\operatorname{MNR}(T)=\int \psi_{T}(s)\left(\left(\theta\left(e^{s}\right)-\Sigma(s)\right) / e^{s / 2}\right) d s
$$

is eventually negative (cf. [6]); here,

$$
\Sigma(s)=\sum e^{\left(1 / 2+\rho_{j}\right) s} /\left(1 / 2+\rho_{j}\right),
$$

the sum is over the exceptional eigenvalues $\lambda_{j}=1 / 4-\rho_{j}^{2}<1 / 4, \rho_{j}>0$, of the Laplacian, $\psi_{T}(s)=\psi(s / T) / T$, and $\psi$ is $C^{(1)}$ with piecewise continuous second derivative and

$$
\psi(s) \geq 0, \quad \operatorname{supp} \psi \subset(0,1) \text { and } \int \psi(s) d s=1 .
$$

In the case of $\Gamma(2), \Sigma(s)=e^{s}$. A similar result holds for the prime geodesic function $\pi(x)$ (see Theorem 4.3).

Theorem 4.1. Let $\Gamma$ be a discrete cofinite subgroup of $\operatorname{PSL}(2, R)$ with $\kappa>0$ cusps and $\nu$ cusp forms at $\lambda=1 / 4$. Then

$$
\lim _{T \rightarrow \infty} \operatorname{MNR}(T)=4 \nu-\kappa+\operatorname{tr}(\Phi(1 / 2))-1,
$$

where $\Phi$ is the scattering matrix.

Remark 1. If $\Gamma$ is cocompact, then $\kappa=0=\Phi$. 
Remark 2. For $\Gamma(2), \kappa=3, \nu=0$, and $\operatorname{tr}(\Phi(1 / 2))=-3$, so that the limit is equal to -7 .

Proof of Theorem 4.1. We begin by setting

$$
\omega_{s}(\sigma)= \begin{cases}e^{|\sigma| / 2} & \text { for }|\sigma|<s, \\ 0 & \text { for }|\sigma| \geq s,\end{cases}
$$

and

$$
g_{T}(\sigma)=\int_{0}^{T} \psi_{T}(s) \omega_{s}(\sigma) e^{-s / 2} d s
$$

Then

$$
\theta\left(e^{s}\right)=\sum \ln (N(\gamma)) N(\gamma)^{-1 / 2} \omega_{s}(\ln (N(\gamma)))
$$

and

$$
\int_{0}^{T} \psi_{T}(s) \theta\left(e^{s}\right) e^{-s / 2} d s=\sum \ln (N(\gamma)) N(\gamma)^{-1 / 2} g_{T}(\ln (N(\gamma))),
$$

both sums being over the primitive conjugacy classes $\{\gamma\}$ of $\Gamma$. The integral $g_{T}$ in (4.6) is an even function with support in $(-T, T)$ and

$$
g_{T}(0)=c(T)=\int_{0}^{1} \psi(u) e^{-u T / 2} d u=O(1 / T) .
$$

The Fourier transform of $g_{T}$ is

$$
\begin{aligned}
h_{T}(r) & =2 \int_{0}^{T} \cos \sigma r g_{T}(\sigma) d \sigma=2 \int_{0}^{T} \psi_{T}(s) e^{-s / 2}\left(\int_{0}^{s} \cos \sigma r e^{\sigma / 2} d \sigma\right) d s \\
& =4\left(1+4 r^{2}\right)^{-1}\left(\int_{0}^{1}(\cos \sigma r T+2 r \sin \sigma r T) \psi(\sigma) d \sigma-c(T)\right) .
\end{aligned}
$$

Upon integrating the right-hand side by parts, we see that

$$
h_{T}(r)=O\left(1 /\left(T r^{2}\right)\right)
$$

and is uniformly bounded. Similarly,

$$
h_{T}^{\prime}(r)=O\left(1 /\left(\left(1+r^{2}\right)|r|\right)\right)
$$

and in addition, $h_{T}^{\prime}(r) \rightarrow 0$ for each $r$ as $T \rightarrow \infty$. It is easily verified that

$$
h_{T}(0)=4(1-c(T)) \rightarrow 4
$$

and by $(4.10)$ that

$$
\begin{aligned}
h_{T}(i \rho) & =\int_{0}^{T} \psi_{T}(s) e^{-s / 2}\left(\int_{0}^{s}\left(e^{\sigma \rho}+e^{-\sigma \rho}\right) e^{\sigma / 2} d \sigma\right) d s \\
& =\int_{0}^{T} e^{\rho s}(1 / 2+\rho)^{-1} \psi_{T}(s) d s+O(1 / T) .
\end{aligned}
$$


Summing over the exceptional eigenvalues $\lambda_{j}=1 / 4-\rho_{j}^{2}<1 / 4, \rho_{j}>0$, we get

$$
\sum h_{T}\left(i \rho_{j}\right)=2 \int_{0}^{T} \psi_{T}(s) \sum(s) e^{-s / 2} d s+O(1 / T) .
$$

In order to apply the Selberg trace formula, we first have to mollify $\omega_{s}$. To this end, let $\zeta(\sigma) \in C^{\infty}(R)$ have support in $(-1,1)$ with $\int \zeta(\sigma) d \sigma=1$ and set $\zeta_{\varepsilon}(\sigma)=\zeta(\sigma / \varepsilon) / \varepsilon$. Replacing $\omega_{s}$ by $\omega_{s} \star \zeta_{\varepsilon}$, we now deal with

$$
g_{T, \varepsilon}(\sigma)=g_{T} \star \zeta_{\varepsilon} \quad \text { and } h_{T, \varepsilon}(r)=h_{T}(r) \tilde{\zeta}_{\varepsilon}(r) .
$$

The Selberg trace formula [10] can now be written as

$$
\begin{aligned}
\sum h_{T, \varepsilon}\left(r_{j}\right)+\sum h_{T, \varepsilon}\left(i \rho_{j}\right)+2 \nu h_{T, \varepsilon}(0)-1 / 2 \pi \int h_{T, \varepsilon}(r) \frac{\varphi^{\prime}}{\varphi}(1 / 2+i r) d r \\
=|F| / 2 \pi \int h_{T, \varepsilon}(r) r \tanh (\pi r) d r+1 / 2[\kappa-\operatorname{tr}(\Phi(1 / 2))] h_{T, \varepsilon}(0) \\
\quad-2 \kappa \ln 2 g_{T, \varepsilon}(0)+\text { elliptic + hyperbolic }-\kappa / \pi \int h_{T, \varepsilon}(r) \frac{\Gamma^{\prime}}{\Gamma}(1 / 2+i r) d r,
\end{aligned}
$$

where $\varphi=\operatorname{det}(\Phi)$.

To within $o(1)$, the sum over the real nonzero $r$ 's together with the integral on the left-hand side is cancelled out by the first term on the right-hand side. In fact, it is known that [10] (cf. [3])

$$
N(R)=\#\left\{\left|r_{j}\right| \leq R\right\}-1 / 2 \pi \int_{-R}^{R} \frac{\varphi^{\prime}}{\varphi}(1 / 2+i r) d r=(|F| / 2 \pi) R^{2}+O(R \ln R),
$$

and it follows from this that the three terms cancel each other to within an error which, by $(4.11)^{\prime}$, is of order

$$
\left|h_{T}^{\prime}(r)\right| r \ln r d r=o(1) .
$$

All of the other terms are finite and remain so as we take the limit as $\varepsilon \rightarrow 0$. We are then left with a trace formula in $g_{T}$ and $h_{T}$ without the above three terms. The $g_{T}(0)$ term, the elliptic term, and the $\int h_{T}(r) \frac{\Gamma^{\prime}}{\Gamma}(1 / 2+i r) d r$ term are easily seen to be of $o(1)$. There remains the sum over the imaginary $r$ 's on the left-hand side (given by $(4.14)$ ), the $h_{T}(0)$ terms and the hyperbolic term on the right-hand side.

The hyperbolic term is now equal to

$$
\text { hyperbolic }=2 \int_{0}^{T} d s \psi_{T}(s) e^{-s / 2} \sum \sum \ln (N(\gamma))\left(1-N(\gamma)^{-k}\right)^{-1},
$$

where the inner sum is over $1 \leq k \leq s / \ln (N(\gamma))$ and the outer sum is over the primitive conjugacy classes with $\ln (N(\gamma)) \leq s$. Hence, the right-hand side of (4.19) can be rewritten as

$$
2 \int_{0}^{T} d s \psi_{T}(s) e^{-s / 2} \sum \theta\left(e^{s / k}\right)+O\left(\int_{0}^{T} d s \psi_{T}(s) e^{-s / 2} \sum \int_{0}^{s} e^{-k \sigma} d \theta\left(e^{\sigma}\right)\right),
$$


where the sums are over $1 \leq k \leq s / m$, with $m=\ln$ (smallest norm). Using the crude estimate $\theta\left(e^{s}\right) \sim e^{s}$, we can easily see that except for the first two terms (in the first sum), this is $O(1 / T)$. The first term is

$$
2 \int_{0}^{T} \psi_{T}(s) e^{-s / 2} \theta\left(e^{s}\right) d s
$$

whereas the second term converges to 2 as $T \rightarrow \infty$. Combining all of the above (in particular, (4.12), (4.14), (4.16), and (4.20)), we finally end up with the assertion (4.4) as desired.

Since the maximum deviation is clearly greater than the mean deviation, Theorem 4.1 implies the following omega result:

Corollary 4.2. If $4 \nu-\kappa+\operatorname{tr}(\Phi(1 / 2)) \neq 1$, then

$$
\left|\theta\left(e^{s}\right)-\Sigma(s)\right|=\Omega\left(e^{s / 2}\right) .
$$

This approach carries over to the prime geodesic function

$$
\pi(x)=\sum 1
$$

summed over the primitive conjugacy classes of norm $\leq x$. It is clear from the definition of $\theta(x)$ that

$$
\pi\left(e^{s}\right)=\int_{0}^{s} 1 / \sigma d \theta\left(e^{\sigma}\right)=\theta\left(e^{s}\right) / s+\int_{\delta}^{s} \theta\left(e^{\sigma}\right) / \sigma^{2} d \sigma,
$$

where $0<\delta<\min \ln (N(\gamma))$ over all norms $N(\gamma)$ with $\gamma$ hyperbolic. Setting

$$
\Sigma_{1}(s)=\Sigma(s) / s \text { and } \Sigma_{2}(s)=\int_{\delta}^{s} \Sigma(\sigma) / \sigma^{2} d \sigma
$$

we can write

$$
\pi\left(e^{s}\right)-\Sigma_{1}(s)-\Sigma_{2}(s)=\left(\theta\left(e^{s}\right)-\Sigma(s)\right) / s+\int_{\delta}^{s}\left(\theta\left(e^{\sigma}\right)-\Sigma(\sigma)\right) / \sigma^{2} d \sigma .
$$

Theorem 4.3. The mean normalized remainder is given by

(4.26) $\lim _{T \rightarrow \infty} \int_{0}^{T} \psi_{T}(s) s e^{-s / 2}\left(\pi\left(e^{s}\right)-\Sigma_{1}(s)-\Sigma_{2}(s)\right) d s=4 \nu-\kappa+\operatorname{tr}(\Phi(1 / 2))-1$.

Idea of proof. The assertion (4.26) will follow from Theorem 4.1 if we can show that

$$
I(T)=\int_{0}^{T} \psi_{T}(s) s e^{-s / 2} \int_{\delta}^{s}\left(\theta\left(e^{\sigma}\right)-\Sigma(\sigma)\right) / \sigma^{2} d \sigma d s \rightarrow 0 .
$$

To this end, we define $\omega \in C^{\infty}(R)$ as

$$
\omega(\tau)=\left\{\begin{array}{ll}
e^{|\tau| / 2} & \text { for } \delta \leq|\tau|, \\
0 & \text { for }|\tau| \leq \delta / 2
\end{array} \quad \text { and } \quad \omega_{\sigma}(\tau)= \begin{cases}\omega(\tau) & \text { for }|\tau| \leq \sigma \\
0 & \text { for }|\tau|>\sigma\end{cases}\right.
$$


Then

$$
\int_{\delta}^{T} \theta\left(e^{\sigma}\right) / \sigma^{2} d \sigma=\sum \ln (N(\gamma)) N(\gamma)^{-1 / 2} \int_{0}^{T} \omega_{\sigma}(\ln (N(\gamma))) / \sigma^{2} d \sigma,
$$

summed over the primitive conjugacy classes.

Next, we apply the Selberg trace formula, with

$$
\begin{aligned}
g_{T}(\tau)= & \int_{0}^{T} \psi_{T}(s) s e^{-s / 2} \int_{0}^{s} \omega_{\sigma}(\tau) / \sigma^{2} d \sigma d s \\
= & \omega(\tau) \int_{\tau}^{T} \psi_{T}(s) s e^{-s / 2}(1 / \tau-1 / s) d s \\
& h_{T}(r)=2 \int_{0}^{\infty} \cos \tau r g_{T}(\tau) d \tau
\end{aligned}
$$

It is easy to show that $g_{T}(0)=0=g_{T}(T)$ and that

$$
\int_{0}^{T}\left|g_{T}^{(i)}(\tau)\right| d \tau=O(1 / T) \text { and } \int_{0}^{T}\left|\tau g_{T}^{(i)}(\tau)\right| d \tau=O(1) \quad \text { for } i=0, \ldots, 3
$$

and it follows from this that

$$
h_{T}(r)=O\left(1 /\left(T\left(1+r^{2}\right)\right)\right) \quad \text { and } \quad h_{T}^{\prime}(r)=O\left(1 /\left(1+|r|^{3}\right)\right)
$$

for the latter estimate we need one more degree of smoothness in $\psi$. Rewriting $h_{T}(r)$ as in (4.10), we can show that $h_{T}^{\prime}(r)=o(1)$ for each $r$. The rest of the argument follows that of Theorem 4.1 .

Corollary 4.4. If $4 \nu-\kappa+\operatorname{tr}(\Phi(1 / 2)) \neq 1$, then

$$
\left|\pi\left(e^{s}\right)-\Sigma_{1}(s)-\Sigma_{2}(s)\right|=\Omega\left(s^{s / 2} / s\right) .
$$

In computing the MNR we have used the formula

$$
\operatorname{MNR}(T)=\int_{50}^{T} \text { normalized mean } d \operatorname{tr} /(T-50) .
$$

To make this into a mean of the type used in Theorems 4.1 and 4.3, we would have to change $d \operatorname{tr}$ into $d \operatorname{tr} / \operatorname{tr}$, the integral range into 1 to $T$, and $(T-50)$ into $\ln (T)$. We chose to give more weight to the larger values of $\operatorname{tr}$.

\section{ACKNOWLEDGMENT}

I would like to thank Peter Sarnak for his helpful suggestions and continued interest in this paper.

\section{BIBLIOGRAPHY}

1. A. Aurich, E. B. Bogomolny, and F. Steiner, Periodic orbits on the regular hyperbolic octagon, Phys. D 45 (1991), 91-101.

2. M. V. Berry, Semiclassical theory of spectral rigidity, Proc. Roy. Soc. London Ser. A $\mathbf{4 0 0}$ (1985), 229-251.

3. D. Hejhal, The Selberg trace formula for $\operatorname{PSL}(2, R)$, vol. 1, Lecture Notes in Math., No. 548, Springer-Verlag, Berlin and New York, 1976.

4. H. Iwaniec, Prime geodesic theorem, J. Reine Angew. Math. 349 (1984), 136-159. 
5. W. J. Luo and P. Sarnak, Number variance for arithmetic hyperbolic surfaces, Comm. Math. Phys. 161 (1994), 419-432.

6. R. Phillips and Z. Rudnick, The circle problem in the hyperbolic plane, J. Funct. Anal. 121 (1994), 78-116.

7. R. Phillips and P. Sarnak, The spectrum of Fermat curves, Geom. Funct. Anal. 1 (1991), 80-146.

8. __ Cusp forms for character varieties, Geom. Funct. Anal. 4 (1994), 93-118.

9. C. Schmit, Quantum and classical properties of some billiards on the hyperbolic plane, Chaos and Quantum Physics (M.-J. Giannoni, A. Voros, and J. Zinn-Justin, eds.), NorthHolland, Amsterdam, 1991, pp. 333-369.

10. A. Selberg, Göttingen lectures, Collected works I, Springer-Verlag, Berlin and New York, 1989.

11. S. Wolpert, The disappearance of cusp forms in special families, Ann. of Math. (2) 139 (1994), 239-291.

Department of Mathematics, Stanford University, Stanford, California 94305

E-mail address: rspegauss.stanford.edu 


\section{Supplement to}

\section{CONJUGACY CLASSES OF $\Gamma(2)$ AND SPECTRAL RIGIDITY}

\section{RALPH PHILLIPS}

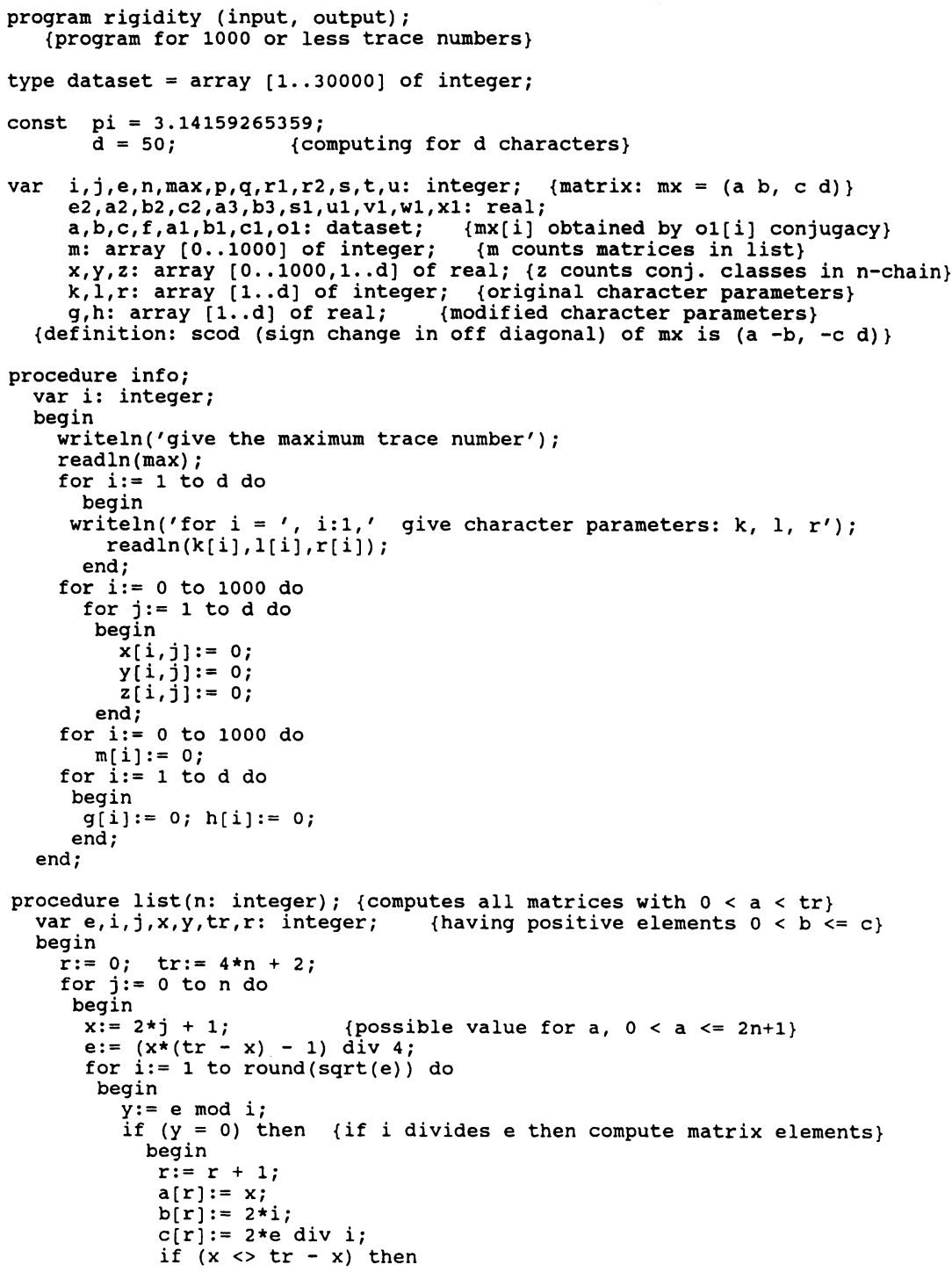



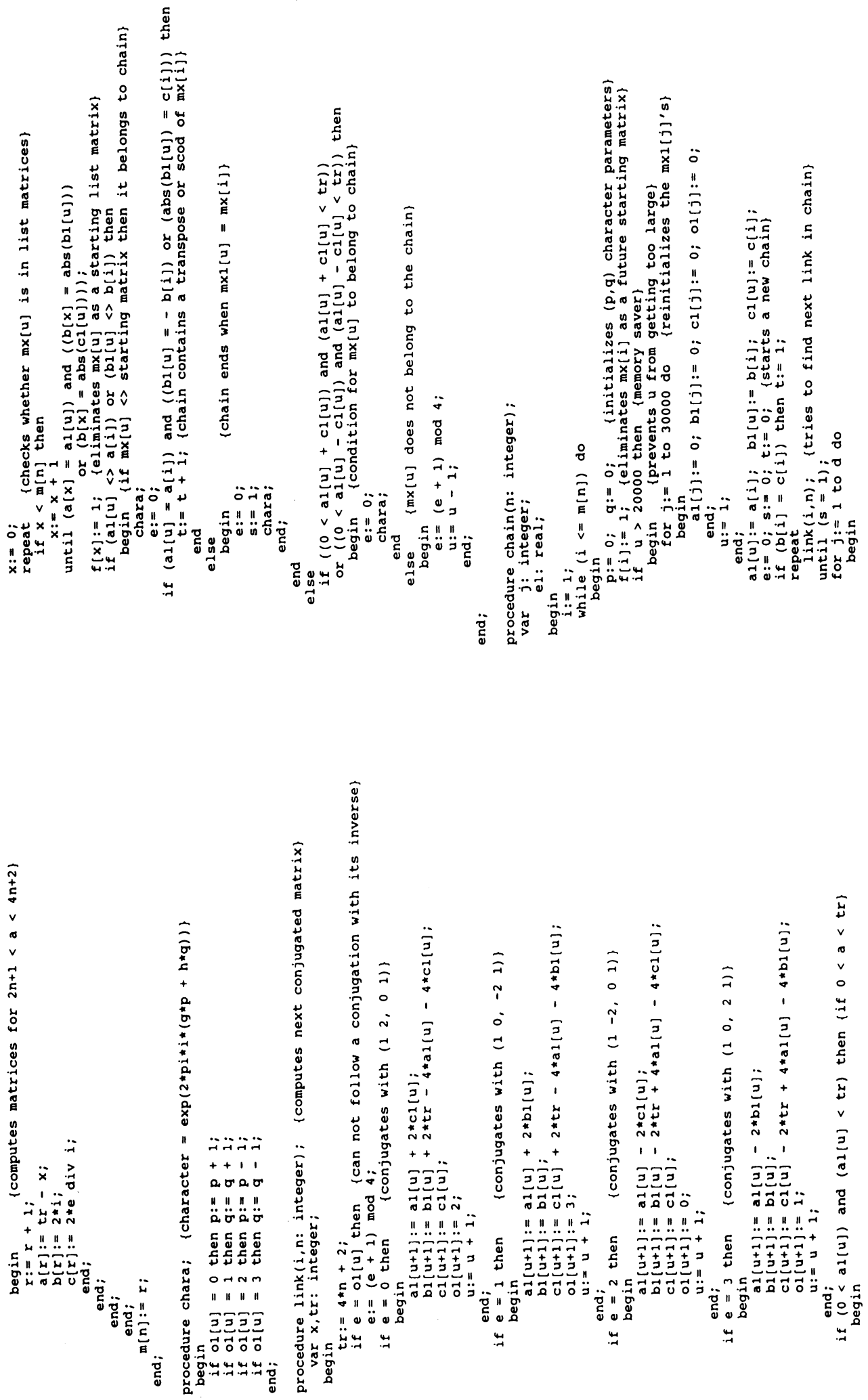

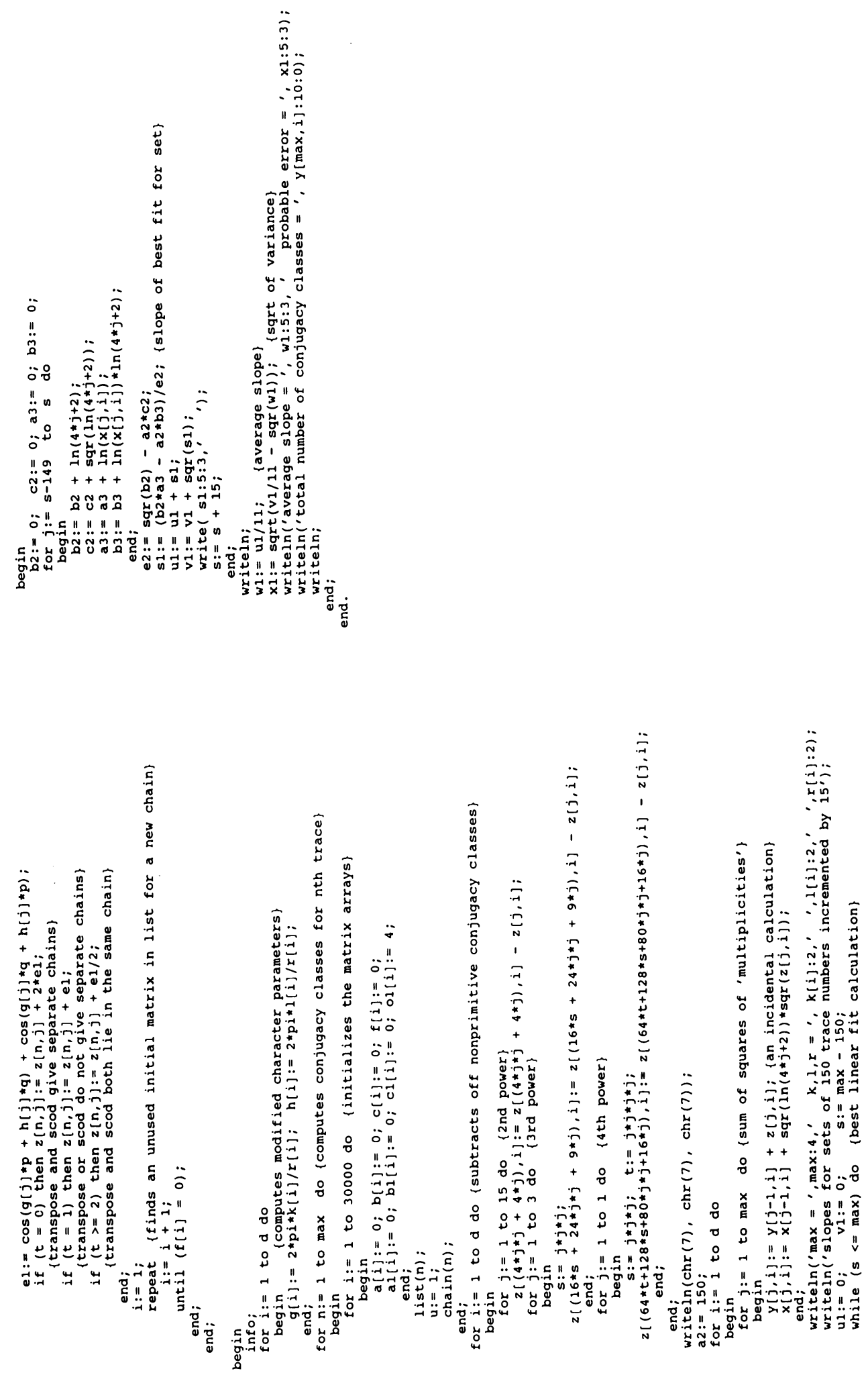